\title{
Cerebral malaria
}

\begin{abstract}
Cerebral Malaria is a potentially reversible encephalopathy caused by Plasmodium falciparum. It is the first cause of death of parasitic origin in the world. There are several theories that seek to explain the pathophysiological mechanisms of the disease, without there being still firm conclusions about it. Clinical manifestations differ between adults and children and their clinical spectrum is very broad. In the treatment, the artemisinin derivatives, artesunate and quinine are the drugs of choice, although the prognosis of the disease does not depend only on its use, since the infinity of associated complications have high repercussion in the same. Current research efforts focus on the development of a vaccine as a preventive measure. The author presents an extensive bibliographic review on Cerebral Malaria, as well as the most relevant aspects for clinical practice and the most controversial ones.
\end{abstract}

Volume 8 Issue 4 - 2018

\author{
Marta Valentim \\ Internal Medicine Department, Hospital Distrital de Santarém, \\ Portugal
}

\section{Correspondence: Marta Valentim, Avenida Bernardo Santareno, 2005- 177 Santarém, Portugal,}

Email marta-m.v@hotmail.com

Received: April 13,2018 | Published: August 03, 2018

Keywords: cerebral malaria, criteria severity, pathophysiology, review

Abbreviations: CM, cerebral malaria; P, Plasmodium; RC, red cells; $\mathrm{BBB}$, blood-brain barrier; $\mathrm{WHO}$, world health organization

\section{Introduction}

Malaria is one of the most prevalent infectious diseases worldwide. ${ }^{1}$ The World Health Organization (WHO) says that $50 \%$ of the world population resides in a country at risk of malaria. It is estimated that there are about 300 million cases and one million annual deaths attributable to it. ${ }^{2-5}$ The growing concern for this disease, not only resides in endemic areas, but is increasingly a concern in non-endemic countries, due to the migratory flow and the ease of traveling. ${ }^{6-8}$ Cerebral Malaria $(\mathrm{CM})$ is the most severe ${ }^{2,9}$ and frequent ${ }^{4}$ complication caused by Plasmodium $(\mathrm{P})$ falciparum infection.

\section{Concept}

$\mathrm{CM}$ is a symmetric and diffuse, potentially reversible encephalopathy caused mainly by $P$. falciparum.$^{4-5,10}$ It is an error to considering that any cerebral clinical manifestation in a patient with malaria is defining the disease ${ }^{3}$ The most agreed definition (Table 1) includes the confirmation of P. falciparum infection and the exclusion of other causes of encephalitis, ${ }^{9}$ since neurological symptoms can be mimicked by metabolic acidosis, anemia or hypoglycemia. ${ }^{2,10,11}$

Table I Criteria for cerebral malaria ${ }^{3}$

\section{Criteria for cerebral malaria}

Positivity for P. Falciparum

Glasgow scale $<$ I I/I 4 in adults or Blantyre $<2$ in children

Exclusion of convulsive postcritical state and hypoglycaemia

Exclusion of other causes of coma (encephalitis, encephalopathies, trauma, toxicity ...)

$\mathrm{CM}$ is not a concept free of conflicts in the literature. The most heated discussion appears when the WHO to define it, uses the term "unarousable coma" (inability to react to the painful stimulus). ${ }^{6}$ Something quite criticized due to the inaccuracy of the concept, ${ }^{9}$ so several authors promulgate the use of the scale of Glasgow was much more widespread and universalized in substitution, pointing out that it would be appropriate to consider a score of 11 or less. ${ }^{9,11}$

\section{Epidemiology}

Malaria is one of the main public health problems in endemic areas, with 300 to 500 million cases and 1 to 1.5 million annual deaths. ${ }^{4-5,11}$ It is the first cause of death, of parasitic origin in the entire world. ${ }^{4}$

P. falciparum is responsible for $80 \%$ of fatal cases hospitalized in endemic areas ${ }^{4}$ and neurological complications are found in $50 \%$ of children admitted to the hospital. ${ }^{6}$ In 2013, an incidence of malaria was estimated to be around 198 million cases worldwide and some 575,000 cases of CM were reported, with the African child population being the most affected, ${ }^{11}$ with a mortality of $25-30 \% 10$.

12,000 cases are considered imported from Europe to Malaria, France being the country with the largest number, about 5,000 cases per year. ${ }^{5}$ In one of the retrospective studies conducted in French intensive care centers, where 400 cases of imported severe malaria were received, a mortality of $10 \%$ was observed. ${ }^{5}$ Imported malaria has an estimated mortality of $10-15 \% .{ }^{5} \mathrm{CM}$ occurs in $2.4 \%$ of travelers with malaria to P. falciparum. ${ }^{6,7}$

\section{Etiology'}

Anopheles: The bite of the mosquito of the female Anopheles spp. Species infected with $P$. falciparum transmits the disease to the human being. ${ }^{2,11}$ The importance of these vectors lies in being one of the objectives of the malaria control programs. ${ }^{12}$ In the mosquito life cycle we distinguish 4 stages: egg, larva, chrysalis and adult. All these phases depend on temperature and nutritional factors, and there is a greater development if temperatures are high. ${ }^{13}$

Plasmodium: Malaria is caused by a group of parasites belonging to the genus Plasmodium spp, ${ }^{2,13}$ which are protozoa of the Sporozoa class, of the order Eucoccidiida and suborder Haemosporidiida. ${ }^{13}$ There are more than 100 species, although only four of them are responsible for Malaria in the human: P. Ovale, $P$. Malariae, $P$. Falciparum and $P$. Vivax. ${ }^{2,14}$ The latter two, being responsible for the majority of cases worldwide.,13 Within the species responsible for more complications, $P$. falciparum ${ }^{4}$ stands out, with $1 \%$ of severe complications that culminated in multi-organ failure. ${ }^{6,14} P$. vivax is responsible for convulsions in children, and also for coma in children and adults. ${ }^{6}$ 
Plasmodium vital Cycle: ${ }^{15}$ In the salivary glands of the female of the Anopheles mosquito are the Sporozoites, that is, the infective form of Plasmodium. The female of the mosquito inoculates by means of a bite, from 5 to 50 Sporozoites to the human being, reaching them into the bloodstream. ${ }^{2,16}$

It is at the arrival to the liver, within the hepatocytes, where the Sporozoites begin their asexual division of the parasite originating the so-called Merozoites and their grouping in Schistocytes. This process of the cycle is called the hepatic phase, where there are no clinical manifestations. ${ }^{2,16}$

With maturation, the hepatocytes are broken, releasing to the circulation all the parasitic forms, where the Merozoites in the socalled hematic phase infect the red cells (RC) developing an annular form, which is the one observed in the blood smear and is considered diagnostic of the disease, the so-called trophozoites. ${ }^{2,16}$ It is the blood phase that is responsible for the pathogenesis of the disease, and therefore the symptoms develop 10 to 15 days after the initial bite. ${ }^{2}$ Replication within the $\mathrm{RC}$ also occurs in a process similar to that occurring in hepatocytes, causing the rupture of these cells when maturation and infection of new $\mathrm{RC}^{2,16}$ have occurred.

During the blood phase, a subpopulation of Merozoites are transformed into Gametocytes, on the $10^{\text {th }}$ to $20^{\text {th }}$ day of infection of the human being. In this phase the mosquito of the female of Anopheles feeds on the blood of the infected human, closing the cycle. ${ }^{2,16}$ In the last stages of the infection, the parasitized red cells (PRC) adhere to the endothelial cells of the venules, in order to promote the growth of the parasite in a somewhat hypoxic environment in order to prevent its destruction by the spleen. ${ }^{6}$

\section{Fisiopathology ${ }^{17}$}

There is no consensus on the pathogenesis of $\mathrm{CM},{ }^{11}$ since studies have been hampered by the difficulty in obtaining brain samples., ${ }^{2,18}$ Efforts to elucidate the mechanisms have focused on the use of models with mice in vivo and post-mortem studies of brain autopsy tissue from endemic areas. ${ }^{11}$

Among the anatomo-pathological findings of the brain biopsies of patients with $\mathrm{CM}$ was:

Macroscopically: cerebral edema with diffuse petechial hemorrhage predominantly in the white matter and malarial pigment in the cerebral cortex.

Microscopically: thickening of capillaries and cerebral venules with PRC and RC. Endothelium and blood-brain barrier (BBB) damage is found by immunohistochemistry. In later phases, we speak about the granuloma of Dürck formed by glial reactions and cellular immunological response in the areas of vascular necrosis of the CNS

Due to these descriptions the importance of BBB was discovered, although the parasites are not able to cross it, they generate a damage of the $\mathrm{BBB}$ and an associated hemorrhage that allows the parasitic advance $^{2}$

There is a physiopathological model described in 4 phases: ${ }^{4-6,8,11,18-22}$

i. Cytoadherence and sequestration of PRC in the cerebral microvasculature

ii. PRC agglutination iii. Production of cytokines and activation of second messengers

iv. Opening of the BBB

In 2002, a model was published by Adams and his collaborators ${ }^{23}$ that could explain the changes suffered by the BBB, responsible for the clinic not only observable in $\mathrm{CM}$, but also in other encephalopathies. The action of cytokines and adhesion processes at the level of endothelial cells generate the activation of signaling pathways in the $\mathrm{BBB}$. When the FAK is activated, the alteration of the cytoskeleton occurs, increasing the permeability of the BBB. This increase in permeability will cause the passage of plasma proteins to the CNS that will generate edema due to oncotic pressure variations and the entry of cytokines with inflammatory cascade activation, which is doubtful whether it will be the mechanism responsible for the neurological damage.

Other theories that explain brain injury are: ${ }^{14,21}$

Permeability hypothesis: toxic substances generated by the parasite increase the permeability of the BBB, generating edema, coma and death. In contrast, increased intracranial pressure is not common in adults and there is no response to corticosteroids.

Cytokine hypothesis: in the breakdown of the merozoites a glycolipid similar to an endotoxin is released, which induces the production of cytokines. In favor of this theory, the high concentration of TNF alpha in serum of patients with CM.

Mechanical hypothesis: the genesis of an obstruction by the PRC in the cerebral circulation could explain the coma.

\section{Clinical manifestations ${ }^{14,22,24}$}

The first symptoms usually occur 10 to 15 days after the bite and include as main manifestations: high fever $\left(40^{\circ} \mathrm{C}-40.5^{\circ} \mathrm{C}\right)$, myalgias, headaches, sweating and other typical manifestations of a flu syndrome..$^{2-3,6}$

In children and immunosuppressed the first manifestations can occur the first 6 to 12 hours. ${ }^{3}$ Hypothermia is a rare manifestation, but observable in cases of shock. ${ }^{3}$ Other reported manifestations are: nausea, vomiting, abdominal pain, tachycardia, arthralgias, hepatosplenomegaly. ${ }^{3,6}$

i. SERIOUS MALARIA CRITERIA: $:^{3-5,11,14}$ It is important to intensificated the surveillance and monitoring (Table 2).

ii. NEUROLOGICAL ALTERATIONS: Plasmodium in the brain, is responsible for a symmetric encephalopathy with lesion of the upper motor neuron, ${ }^{4,14}$ which is why, it has a wide spectrum of manifestations: from headaches to deterioration of the level of consciousness. $^{2,6}$

Coma: ${ }^{25}$ the cerebral blood flow is inappropriately low, creating an anaerobic environment at the brain level, which prduces an increase in lactate concentration in the CSF interfering with neurotransmission. ${ }^{14}$ In patients in coma or febrile it is common to find a divergent view with reactive pupils and normal oculocerebral and oculovestibular reflexes. $^{4}$

Seizures: focal or generalized and more frequent in children (70\%) compared to adults $(20 \%))^{3-4,6}$ Although the majority of seizures seem to be generalized in clinical observation, it is the EEG that identifies most of them with a focal origin of the same, ${ }^{6}$ as well as alterations 
in the EEG without clinical translation are common. ${ }^{6}$ Seizures are associated with an increase in intracranial pressure. ${ }^{6}$ Epileptic status is rare in CM..$^{3-4,6}$ In adults, seizures can occur as part of a postmalarial neurological syndrome and in children after $\mathrm{CM}$ recovery6.

Ocular manifestations: retinal hemorrhage (RH), called "in flame", is present in $15 \%$ of adult patients ${ }^{3}$ and up to $40 \%$ in children, ${ }^{4}$ and strongly correlates with a poor prognosis. ${ }^{4}$ It is described, a relationship between the number of HR and the number of white matter hemorrhages were found in the necropsies of patients with CM. ${ }^{4}$ Photophobia and papilledema is rare. ${ }^{3-4,6}$

Other rare alterations: meningism, signs of neurological focality and neuroophthalmological signs are described. ${ }^{3,6}$

i. Osteotendinous reflexes: the manifestations vary from increased ROTs, highlighting the aquileo reflex, up to areflexia. ${ }^{4,6}$

ii. Cutaneous reflexes: normally absent indicating alterations of the pyramidal system. ${ }^{4}$

iii. Oculocephalic and oculovesticular reflexes: they are usually altered in children. ${ }^{6}$

iv. Nuchal rigidity: it is frequent in adults, simulating a meningeal syndrome. Although it is considered a rare manifestation. ${ }^{4}$

v. Psychomotor agitation: rare manifestation, simulating intoxication or an episode of acute psychosis. ${ }^{4}$

vi. Decortication and decerebration: it is more common in children than in adults. These positions can occur in association with hypoglycemia, but should, in most cases, lead us to suspect an increase in intracranial pressure. ${ }^{6}$

vii. Alterations circadian rhythm and insomnia. ${ }^{6}$

Psychiatric manifestations: hallucinations, psychosis or illusions, may be symptoms that the patient experiences in the recovery phase. Likewise, they may be due to the side effects of antimalarial medication. ${ }^{6,26}$

Table 2 Criteria for complicated malaria (adapt from WHO classification)

\begin{tabular}{|c|c|}
\hline Clinical criteria & Lab criteria \\
\hline $\begin{array}{l}\text { Altered state of } \\
\text { consciousness: GCS } \\
\text { less than II. }\end{array}$ & $\begin{array}{l}\text { Severe anemia: normochromic normochromic } \\
\text { anemia with a hematocrit of less than } 15 \% \text { or a } \\
\text { hemoglobin of less than } 5 \mathrm{~g} / \mathrm{dL} \text { in the presence } \\
\text { of parasitism above } 1000 / \text { microlitres. }\end{array}$ \\
\hline $\begin{array}{l}\text { Pulmonary edema/ } \\
\text { ARDS }\end{array}$ & $\begin{array}{l}\text { Renal failure: diuresis less than } 400 \mathrm{~mL} \text { in } \\
24 \text { hours in adults or less than } 12 \mathrm{~mL} / \mathrm{kg} \text { in } \\
\text { children. In this type of kidney injury there is no } \\
\text { improvement despite rehydration. There is talk } \\
\text { of serum creatinine levels higher than } 3 \mathrm{mg} / \mathrm{dL} \text {. }\end{array}$ \\
\hline $\begin{array}{l}\text { Macroscopic } \\
\text { hemoglobinuria }\end{array}$ & Hypoglycemia: levels below $40 \mathrm{mg} / \mathrm{dL}$. \\
\hline $\begin{array}{l}\text { Several episodes of } \\
\text { convulsion. }\end{array}$ & $\begin{array}{l}\text { Acidosis: arterial } \mathrm{pH} \text { less than } 7.25 \text {, bicarbonate } \\
\text { levels less than } 15 \mathrm{mmol} / \mathrm{L} \text {. }\end{array}$ \\
\hline $\begin{array}{l}\text { Shock/hypotensio/signs } \\
\text { of poor perfusion. }\end{array}$ & $\begin{array}{l}\text { Hyperparasitaemia: in immunocompetent } \\
\text { individuals, levels higher than } 10 \% \text { or higher } \\
\text { than } 5 \% \text { in immunocompromised patients. }\end{array}$ \\
\hline Hemorrhages. & Hyperthermia: rectal temperature above $40^{\circ} \mathrm{C}$. \\
\hline $\begin{array}{l}\text { Jaundice/bilirubin more } \\
\text { than } 3 \mathrm{mg} / \mathrm{dL} \text {. }\end{array}$ & Lactate levels greater than $5 \mathrm{mmol} / \mathrm{L}$ \\
\hline
\end{tabular}

\section{Diagnostic}

The diagnostic of CM should be considered in all patients with decreased state of consciousness, with a history of fever, who has been in an endemic area for 2 to 3 months before the onset of symptoms. ${ }^{6,27}$ Occasionally, there are cases described in residents near airports or who have had contact with blood products. ${ }^{6}$ To make the diagnosis, the initial approach requires the presence of standard criteria (Table 3). ${ }^{4}$

Table 3 Diagnostic criteria ${ }^{4}$

\section{Diagnostic criteria}

Altered state of consciousness or coma (GCS scales in adults or Blantyre in children). If it occurs with or without seizures, the coma should persist for about 6 hours after the seizure episode.

Exclusion of other encephalopathies or conditions that can mimic the clinic of CM. ${ }^{15}$

Positivity for Plasmodium falciparum in thick blood.

The diagnosis must be confirmed (thick and thin microscopy with $1000 \%$ magnification and Giemsa stain), ${ }^{3,27}$ after which the species and parasitemia will be identified. ${ }^{27}$ Situations that can lead to false negatives are the initial phases of treatment with antimalarials or the sequestration of PRC in the cerebral circulation. ${ }^{3}$ In these situations we must repeat the smear every 12 hours during the first 48 hours, before assuming another diagnosis., 3

Other techniques used are PCR and rapid qualitative tests for the detection of antigens that detect PfHRP2 and pLDH of P. falciparum, have a diagnostic sensitivity similar to the method by microscopy without the need of an experienced microscopist. ${ }^{3,27}$ Keep in mind that PfHRP2 continues to circulate weeks after the cure, which can cause false positives. ${ }^{27}$ The reality about these techniques is the difficulty in their application and the little interest they have aroused in the field. ${ }^{28}$

Neuroimaging exams are useful to exclude other pathologies. ${ }^{29}$ Both CT and MRI describe nonspecific findings (15-20\%) such as: cerebral edema, ${ }^{1,30}$ cortical infarcts and hyperintense areas in white matter. ${ }^{3-4,6,29}$ The examination of the fundus is useful ${ }^{31}$ to detect retinal hemorrhages, which correlate with brain damage., ${ }^{4,32}$

Lumbar puncture should be performed to exclude other causes of febrile syndromes with involvement of the state of consciousness. ${ }^{6,27,33}$ Normally, the CSF presents a pleocytosis of 10 to 50 cells $/ \mathrm{mm}^{3}$ with a high protein level of $200 \mathrm{mg} / \mathrm{dL} .{ }^{3,6}$ Even in the pediatric population the need for its realization is clear, despite this in some African pediatric centers, the decision is postponed due to the probability of herniation related to the increase in intracranial pressure, present in most cases; starting antibiotic treatment directly. ${ }^{27,33}$

The EEG shows nonspecific alterations 3 such as: diffuse lentification, pattern of spikes and a pattern of burst suppression. ${ }^{3}$ Post mortem diagnosis by brain biopsy is little reported in the literature. It has been described that in the gray matter, erythrocytes contain the mature forms of schistocytes and trophozoites. ${ }^{14}$ Differential diagnoses of $\mathrm{CM}$ include: bacterial meningitis and tuberculous meningoencephalitis. ${ }^{3}$ For this the realization of lumbar puncture can help to differentiate them. ${ }^{4}$ 


\section{Treatment}

$\mathrm{CM}$ is a neurological emergency that requires urgent intervention, therefore the indicated place for these patients is the intensive care unit, where can be provided: monitoring, surveillance and support treatment. $^{3-5,11}$ In endemic areas, treatment should be started without having the confirmation of the diagnosis.

\section{Pharmacological treatment against the agent $\mathrm{t}^{1,8,34}$}

The intravenous administration of artemisinin derivatives, artesunate and quinine are the drugs of choice. Chloroquine has been abandoned due to resistances. ${ }^{3,6}$ It has been described that artesunate reduces mortality by $34.7 \%$ compared to quinine, in addition to reducing convulsive episodes, coma and hypoglycemia.,

Quinine has a narrow therapeutic window and only has activity on the parasites in the last stages of the erythrocyte cycle. ${ }^{6}$ It can induce hypoglycemia by promoting insulin secretion. ${ }^{6}$ Rapid administration intravenously can cause hypotension. ${ }^{6}$ Another effect is the prolongation of the QT interval, so there must be tight monitoring and should be suspended if the interval exceeds $25 \%$ of the base value of the patient. ${ }^{3,6}$

Artesunate has low toxicity, ease of administration and greater efficacy, since it acts in all stages of the parasitic cycle, it is therefore considered the drug of choice, ${ }^{3,6}$ without the effects described for quinine. $^{3}$

Both drugs are usually combined in order to shorten the duration of treatment and prevent resistance. ${ }^{6}$ So it is possible to give priority to the oral route. ${ }^{3}$ In case of renal or hepatic dysfunction, the doses should be adjusted in contrast to patients on hemodialysis or hemofiltration. ${ }^{3}$

\section{Management of complications"}

The neurological complications of malaria, are usually associated with dysfunction of other organs, with effects such: severe anemia, hypoglycaemia, acute renal failure, liver failure and coagulation disorders. ${ }^{6}$

Hypoglycemia: occurs in 3\% of adults with severe falciparum malaria, and is particularly common in children, pregnant women, and patients receiving quinine therapy. Hypoglycemia often has no obvious clinical signs, so blood glucose levels should be monitored. It responds well to standard therapy, although hyperinsulinemic hypoglycaemia in association with quinine therapy responds well to long-acting somatostatin analogues. ${ }^{6}$

Convulsions: respond to benzodiazepines, although these drugs may be less effective, since malaria seems to decrease the regulation of $\gamma$-aminobutyric acid receptors. Prolonged seizures may require phenobarbital or phenytoin6. Although phenobarbital reduces seizures, mortality in children increases. ${ }^{3}$

Hydro-electrolyte balance: ${ }^{35}$ Fluid balance is critical in severe malaria. In children there is no consensus, and in adults can develop renal failure and/or pulmonary edema, so fluids should be restricted. There is no consensus in patients with CM. Ionic disturbances occur frequently, but are rarely associated with clinical manifestations.

Severe anemia: In adults, a smaller amount of blood is given due to fluid overload. WHO recommends that an exchange transfusion be considered if the patient has severe malaria (deep coma, hypoglycaemia, acidosis or severe anemia) with parasitaemia more than $20 \%$, however, there are no data available that demonstrate improved results with exchange transfusions. ${ }^{3,6}$

\section{Adjuvant treatments ${ }^{36}$}

Many clinical trials studied adjuvant treatments, however, none showed efficacy. ${ }^{3,11,37}$

i. Corticosteroids: they have not shown any benefit, in fact, dexamethasone was associated with an increase in complications (gastrointestinal hemorrhage, sepsis and prolonged coma recovery time). ${ }^{6}$

ii. Desferroxamine: some results showed a faster recovery, others a decrease and a improvement of: seizures, fever and the coma period, however, other studies deny it. ${ }^{6}$

iii. Erythropoietin: it has a neuroprotective role. It has been shown that children with CM and high levels of erythropoietin have a higher probability of surviving without neurological sequelae. ${ }^{6}$

iv. $\mathbf{N}$-acetylcysteine: this drug normalizes serum lactate levels in adults with severe malaria and is currently being tested in adults with $\mathrm{CM}{ }^{6}$

The use of prophylactic anticonvulsants, mannitol, immunoglobulins, albumin, pentoxifylline, heparin, anti-TNF antibodies, does not seem to improve the results of CM.,13,38 There are many doubts to date, since the studies that are available are limited, with small and non-randomized samples.

\section{Prognostic}

$\mathrm{CM}$ has a high mortality, between $15-50 \%{ }^{3-5}$ In the initial approach of the patient there are 3 independent factors associated with mortality mortality: advanced age, presence of coma and elevated parasitism. ${ }^{5}$

The prognosis of $\mathrm{CM}$ depends to a large extent on the management of complications such as renal failure, severe jaundice and metabolic acidosis. ${ }^{4}$ Some articles consider metabolic acidosis in itself, the best predictor of death in severe forms of malaria. ${ }^{19}$ There are several predictors of bad prognosis (Table 4).

Table 4 Factors of poor prognosis in $\mathrm{CM}^{3}$

\section{Factors of poor prognosis in CM}

\section{Recurrent seizures}

Absence of corneal reflex

Decerebration

Retinal hemorrhages

Patients under 3years

High levels of parasitemia $>20 \%$

Leukocytosis in peripheral blood with presence of pigments in polymorphouclears $>5 \%$

Lactic acidosis

CSF lactate elevated

Serum transaminases increased

Low levels of antithrombin III

Genetic factors, both humans and parasites, play an important role in the severity of the disease. ${ }^{11}$ 
Sequelae: ${ }^{39} 90 \%$ of patients with CM recover without neurological sequelae. ${ }^{4}$ The sequelae are more severe in children than in adults, and their prevalence varies between $6-29 \% .^{6}$ Transient neurological sequelae occur in $10 \%-18 \%$ and generally they revert in 4 to 8 weeks. ${ }^{3,6}$

In adults, the spectrum of presentation includes: psychosis (4\%), cerebellar ataxia $(3 \%)$, extrapyramidal rigidity or hemiplegia. ${ }^{40}$ The depth, duration of the coma and multiple seizures were factors for their development. ${ }^{6}$

Cognitive difficulties, language and behavior problems have been documented in $24 \%$ of children after CM. The prevalence of these alterations in adults is not known. ${ }^{6}$ Of the clinical spectrum, psychosis is the most common sequel described, also highlighting: $:{ }^{40}$ hemiplegia, cerebral palsy, blindness and cortical deafness, impairment of cognition and learning. ${ }^{6,14}$ Among the rarest manifestations are cases of Gullain-Barre syndrome, cerebellar ataxia, extrapyramidal symptoms, trismus, peripheral neuropathy, isolated and cranial nerve palsy, nystagmus (vertical and horizontal), blindness due to vitreous hemorrhage and encephalomyelitis. ${ }^{4,14}$

Postmalaria syndrome:26,41 It is defined as the appearance of neurological and psychiatric symptoms about 2 months after the resolution of the malarial infection and where the parasitemia levels are zero. This syndrome is found in $0.1 \%$ of patients with severe malaria criteria and clinically it stands out for the appearance of generalized convulsions, psychosis, tremor or cerebellar ataxia, the latter being the most described in those related to CM. Another described symptomatology included in this syndrome are: myoclonus, aphasia and confusional states. ${ }^{3}$ It should be noted that this is a selflimiting process, ${ }^{6}$ attributable to an immune-mediated process with a response to corticosteroids 3 .

\section{Prevention}

The preventive measures such as the control of the vector or the attempt to break the life cycle of the parasite, ${ }^{42}$ globally used in the tropics are widely known. So far, the greatest failure in the fight against malaria is resistance and the lack of an effective vaccine. ${ }^{11,43-44}$ Resistances are due to genetic mutations that lead to alterations of the targets where the drugs act. ${ }^{11}$ The current approach to the development of a vaccine is based on the use of recombinant proteins or the whole attenuated organism. The vaccines are designed for several stages of the life cycle of the parasite: the pre-erythrocytic for the prevention of infection, the blood phase for the prevention of clinical disease and the vaccines in the sexual phase to block transmission. ${ }^{11}$ The most advanced candidate vaccine for P. falciparum (RTS, S-AS01) is in phase III efficacy trials. ${ }^{11}$

\section{The future}

i. Define the pathophysiology better in order to understand the neurological complications of CM. Currently, MRI is being used to describe structural lesions and electroencephalogram for the characterization of seizures and monitoring of treatment. ${ }^{6}$

ii. Discovery of brain injury markers: ${ }^{6}$ The beta-Amyloid protein is currently relevant. ${ }^{4,11}$

iii. Research into adjuvant therapeutic potentials. ${ }^{6}$

iv. Resistance to therapeutic agents available in endemic areas makes the study of vaccines that are currently not fully developed important. $11,42,44-45$

\section{Acknowledgements}

None.

\section{Conflict of interest}

The author declares no conflict of interest.

\section{References}

1. White NJ, Pukrittayakamee S, Hien TT, et al. Malaria. Lancet. 2014;383(9918):723-375.

2. Laurent Rnia, Howland SW, Claser C, et al. "Cerebral malaria: Mysteries at the blood-brain barrier." Virulence. 2012;3(2):193-201.

3. Misra UK, Kalita J, Prabhakar S, et al. "Cerebral malaria and bacterial meningitis.” Ann Indian Acad Neurol. 2011;14(Suppl1):S35-39.

4. Carlos Hugo Zapata e Sivia Blair Trujillo. "Malaria cerebral". IATREIA. 2003;16(3):202-216.

5. Arben Ndreu, Hajdari D, Ndoni A, et al. Epidemiological, clinical and therapeutic aspects of cerebral malaria imported in Albania. J Infect Dev Ctries. 2016;10(2):190-194.

6. Mishra SK, Newton CR. Diagnosis and management of the neurological complications of falciparum malaria. Nat Rev Neurol. 2009;5(4):189-198.

7. Kalinowska-Nowak A, Bociaga-Jasik M, Leśniak M, et al. The risk of malaria during travel, observations in the department of infectious diseases in Cracow from 1996 to 2010. Przegl Epidemiol. 2012;66(3):431-436.

8. Postels DG, Birbeck GL. Cerebral malaria. Handb Clin Neurol. 2013;114:91-102.

9. Newton CRJC, Pasvol G, Winstanley PA, et al. "Cerebral malaria: what is unarousable coma?” Lancet. 1990;335(8687)472.

10. García López Hortelano M, Fumadó Pérez V, González Toméet MI, et al. "Actualización en el diagnóstico y tratamiento de la malaria." An Pediatr (Barc). 2013;78(2):124.e1-124.e8.

11. Wah ST, Hananantachai H, Kerdpin U, et al. Molecular basis of human cerebral malaria development. Trop Med Health. 2016;44:33.

12. Rubert A, Guillon-Grammatico L, Chandenier J, et al. "Insecticide resistance in Anopheles mosquitoes : additional obstacles in the battle against malaria." Med Sante Trop. 2016;26(4):423-431.

13. Sinka. A global map of dominant malaria vectors." Parasites \& Vectors. 2012;5:69.

14. Dharmeshkumar N Patel, PPradeep, MM Surti, et al. Clinical manifestations of complicated malaria - an overview. JIACM. 2003;4(4):323-31

15. Beri D, Balan B, Tatu U. Commit, hide and escape: the story of Plasmodium gametocytes. Parasitology. 2018;16:1-11.

16. Fleta Zaragozano J. "Paludismo: un grave problema de salud mundial". Medicina Integral. 2001;38(4):167-174.

17. Storm J, Craig AG. Pathogenesis of cerebral malaria-inflammation and cytoadherence. Front Cell Infect Microbiol. 2014;4:100.

18. Gillrie MR, Ho M. Dynamic interactions of Plasmodium spp. With vascular endothelium. Tissue Barriers. 2017;5(1):e1268667.

19. Claire L. Mackintosh, Marsh K. Clinical features and pathogenesis of severe malaria. Trends Parasitology. 2004; 20(12):597-603..

20. Plewes $\mathrm{K}$ et al. Pathophysiology, clinical presentation, and treatment of coma and acute kidney injury complicating falciparum malaria. Curr Opin Infect Dis. 2018;31(1):69-77. 
21. Dvorin JD. Getting your head around cerebral malaria. Cell Host Microbe. 2015;22(5):586-588.

22. Hora R, Kapoor $\mathrm{P}$, Thind $\mathrm{KK}$, et al. Cerebral malaria-clinical manifestations and pathogenesis. Metab Brain Dis. 2016;31(2):225-237.

23. Adams S, Brown H, Turner G. Breaking down the blood-brain barrier: signaling a path to cerebral malaria? Trends Parasitol. 2002;18(8):360366.

24. Ahmad S, Shirazi N, Bhat NK, et al. A hospital-based Retrospective Comparative Study of Complications, outcomes, clinical and laboratory parameters of Malaria with and without neurological involvement Mediterr J Hematol Infect Dis. 2017;9(1):e2017006.

25. Isabelle M Medana, Day NP, Sachanonta N, et al. Coma in fatal adult human malaria is not caused by cerebral oedema. Malaria Journal. 2011;10:267.

26. Nevin RL, Croft AM. Psychiatric effects of malaria and anti-malarial drugs: historical and modern perspectives. Malar J. 2016;15:322.

27. Arjen M Dondorp. "Pathophysiology, clinical presentation and treatment of cerebral malaria" Neurology Asia. 2005;10:67-77.

28. OMS Prueba para el diagnóstico de malaria por P. Falciparum. Rev Panam Salud Publica/Pan Am J Public Health. 1997;1(1).

29. Sanjib Mohanty, Taylor TE, Kampondeni S, et al. Magnetic resonance imaging during life: the key to unlock cerebral malaria pathogenesis? Malar J. 2014; 13:276.

30. Mohanty S, Benjamin LA, Majhi M, et al. Magnetic resonance imaging of cerebral malaria patients reveals distinct pathogenetic processes in different parts of the brain. mSphere. 2017;2(3):e00193-17.

31. Swamy L, Beare NAV, Okonkwo O, et al. Funduscopy in cerebral malaria diagnosis: an international survey of practice patterns. Am J Trop Med Hyg. 2018;98(2):516-519.

32. Chandler Villaverde, Namazzi R, Shabani E. et al. Clinical Comparison of Retinopathy-Positive and Retinopathy-Negative Cerebral Malaria. Am J Trop Med Hyg. 2017;96(5):1176-1184.
33. Moxon CA, Zhao L, Li C, et al. Safety of lumbar puncture in comatose children with clinical features of cerebral malaria. Neurology. 2016;87(22):2355-2362.

34. Abdallah TM, Elmardi KA, Elhassan AH, et al. Comparison of artesunate and quinine in the treatment of severe plasmodium falciparum malaria at Kassala hospital, Sudan. J Infect Dev Ctries. 2014;8(5):611-615.

35. Susanne Helena Hodgson, Brian John Angus. Malaria: fluid therapy in severe disease. BMJ Clin Evid. 2016;2016:0913.

36. Varo R, Crowley VM, Sitoe A, et al. Adjunctive therapy for severe malaria: a review and critical appraisal. Malar J. 2018;17(1):47.

37. Krishna S. Adjunctive management of malaria. Curr Opin Infect Dis. 2012;25(5):484-488.

38. Meremikwu M, Marson AG. Routine anticonvulsants for treating cerebral malaria. Cochrane Database Syst Rev. 2002;(2):CD002152.

39. Oluwayemi IO, Brown BJ, Oyedeji OA, et al. Neurological sequelae in survivors of cerebral malaria. Pan Afr Med J. 2013;15:88

40. Oumkaltoum Taiaa, Amil T, Darbi A, et al. Hemiparesis post cerebral malaria. Pan Afr Med J. 2015;20:1

41. Pace AA et al. A new clinical variant of the post-malaria neurological syndrome. J Neurol Sci. 2013;334(1-2):183-185.

42. Matuschewski $\mathrm{K}$. Vaccines against malaria-still a long way to go. FEBS J. 2017;284(16):2560-2568

43. Mahmoudi S, Keshavarz H. Malaria vaccine development: the need for novel approaches: a review article. Iran J Parasitol. 2018;13(1):1-10.

44. Hviid L, Lavstsen T, Jensen AT, et al. A vaccine targeted specifically to prevent cerebral malaria-is there hope? Expert Rev Vaccines. 2018;17(7):565-567.

45. Dayananda KK et al. Epidemiology, drug resistance, and pathophysiology of Plasmodium vivax malaria. J Vector Borne Dis. 2018;55(1):1-8. 